\title{
Entropy Economic Model of the Company
}

\author{
Tatyana V. Ryzhkova ${ }^{1, *}$ \\ ${ }^{1}$ Plekhanov Russian University of Economics, RU-117997, Moscow, Russia
}

\begin{abstract}
The article discusses the entropy approach to the analysis of corporate financial system on the basis of the financial coefficients and market share price rates. The uncertainty of the corporate financial attractiveness is assessed using the entropy indicator of a random vector. Financial indicators such as Liquidity Financial Ratios, Operating Financial Ratios, Leverage Financial Ratios, Profitability Financial Ratios and Market Ratios serve here as vector components. Discrete entropy models of random vectors as well as differential entropy models for continuous probability distributions were used. It is shown that special probability distributions, approximately coinciding with the basic statistical ones for the first two moments, set the boundary of the maximum differential entropy. The entropy indicators were calculated based on the probability and entropy measures.
\end{abstract}

\section{Introduction}

At the present time economic-mathematical methods and models are efficiently applied in corporate financial controlling for business analysis and forecasting.

The beginning of the information theory was connected with the Claude E. Shannon's fundamental work "A Mathematical Theory of Communication" (1948). As the information theory is a mathematical theory there were various attempts to use it in different sciences. A.N. Kolmogorov determined a close relationship between the theory of probability and the metric theory of functions that enabled to use different types of entropy metrics for the description of any formalized structures [1].

An approach developed on the basis of the information theory concept was called the information theoretical approach or the entropy approach.

Modern economic-mathematical methods are often based on stochastic tools. For instance, the Hurst fractal statistics is used in capital market models. However, the Hurst fractal statistics is a single factor characteristic related only to the value of the stock.

Therefore, the entropy approach can be used as a more general quantitative measure of uncertainty, which can take into account the non-linearity of the modeling process. Still, in practice, a linear paradigm, relying on the central limit theorem, which has limitations, is widely used in economic modeling.

The entropy approach in economic-mathematical modeling has no limitations concerning two-moment distributions that are usually required for the regression analysis, correlation analysis, dispersion analysis as well as factor analysis in finance.

Therefore, the entropy model analysis can be regarded as one of the advanced methods of the corporate financial analysis.

\section{Corporate Financial System Entropy Indicators}

The economic-mathematical modeling deals with the company`s investment attractiveness evaluation. In order to create an entropy model for a corporate financial system lets link an entropy indicator with the financial ratios.

It is well known that financial ratios are an integral part of the financial statement analysis. There are five groups of financial ratios: Liquidity Financial Ratios, Operating Financial Ratios, Leverage Financial Ratios, Profitability Financial Ratios as well as Market ratios. Let's take one ratio from each group of financial ratios.

All the ratios can be described by random variables that possess the value of the above ratios.

Let

$X_{1}$ - the random variable equal to Current Ratio; Ratio;

$X_{2}$ - the random variable equal to Financial Leverage

$X_{3}$ - the random variable equal to Return on Assets (ROI);

$X_{4}$ - the random variable equal to Asset Turnover;

$X_{5}$ - the random variable equal to Share Price Rate of Change (ROC);

To obtain the Share Price Rate of Change indicator, the following calculation is used [3]: 


$$
x_{5}=r_{t_{i}}=\frac{S_{t_{i}}-S_{t_{i-1}}}{S_{t_{i-1}}}
$$

$r_{t_{i}}$ - Share Price Rate of Change (ROC) on the ith day of the calculation period;

$S_{t_{i-1}}$ - Share Closing Price on the (i-1)th day of the calculation period;

$S_{t_{i}}$ - Share Closing Price on the ith day of the calculation period;

Let $\bar{X}\left(X_{1}, X_{2}, X_{3}, X_{4}, X_{5}\right)$ be a random vector that has the following values: $x_{1}, x_{2}, x_{3}, x_{4}, x_{5}$. Then the uncertainty evaluation of the corporate financial attractiveness can be defined as the entropy indicator of the random vector $\bar{X}$.

Let

$$
\begin{gathered}
\text { 1. }\left(\Omega_{i}, A_{i}, P 1\right) \mid i=1,2,3,4-\text { the probability space, } \\
\Omega_{i}-\text { the space of elementary events } \\
\Omega_{i}=\left\{\omega_{i 1}, \omega_{i 2}, \omega_{i 3}, \ldots\right\} . \\
\omega_{i j}-\text { the value of the respective ratio - } X_{i}
\end{gathered}
$$

Assume that the random variables related to the first four Financial Ratios $X \mid i=1,2,3,4$ are discreet and belong to the intervals defined according to the characteristic group type.

If $a_{j} \leq x_{i} \leq b_{j}$, then

$$
\omega_{i j}=\left\{x_{i} \in\left[a_{j}, b_{j}\right]\right\}
$$

Let $A_{i}$ be the class of all subsets $\Omega_{i}$ with the properties of $\sigma$-algebra.

Let $P$ 1be the probability measure as the measures ratio of the above discreet subsets:

$$
\begin{gathered}
\mathrm{P} 1\left(\Omega_{i}\right)=1, \mathrm{P} 1(\varnothing)=0, \\
P 1\left(\bigcup_{i-1}^{m} A_{i}\right)=\sum_{i} P 1\left(A_{i}\right), \text { if } A_{i} \bigcap_{i \neq j} A_{j}=\emptyset, \\
P 1\left(A_{i}\right)=\frac{\operatorname{mes}\left(A_{i}\right)}{\operatorname{mes}\left(\Omega_{i}\right)} .
\end{gathered}
$$

2. $\left(\Omega_{5}, A_{5}, P 2\right) \mid i=5$ - the probability space of the variable $X_{5}$ that is equal to the Share Price Rate of Change (ROC). The variable is usually described by a random continuous variable. In order to provide a numerical entropy analysis financial statement data from 1999-2017 of the biggest Russian companies (Lukoil, Rostelecom, MTS) are used.

On the basis of the above data the values of the random variables $X_{1}, X_{2}, X_{3}, X_{4}, X_{5}$ are calculated.

The value of the random variable $X_{2}$ that describes the financial leverage has remained on the same level of about 0.5 that is acceptable for non-trading companies. In this case let`s assume that $X_{2}$ will not influence the entropy indicator.
The $X_{5}$ values are calculated on the basis of the Moscow Exchange data at selected special intervals. The $X_{5}$ values are determined by time series. In case a rare extreme event affects some of the $X_{5}$ values, such $X_{5}$ values should be excluded from the model, if a generalized assessment of the entropy contribution is required.

Otherwise $X_{5}$ values affected by extreme events should be included in the model to clarify and "emphasize" the entropy contribution. This data analysis methodology was shown in [8].

Assume that the random variable $X_{5}$ is continuous and independent from the random variables $X_{1}, X_{3}, X_{4}$. The random variables $X_{1}, X_{3}, X_{4}$ are dependent variables. Let`s introduce a random vector $\bar{X}^{*}\left(X_{1}, X_{3}, X_{4}\right)$, which describes the structural entropy of the corporate financial ratios.

Let $(\Omega, A, P)$ be the probability space equal to the multiplication of the above probability spaces:

$\left(\Omega_{i}, A_{i}, P 1\right) \mid i=1,3,4$

$\Omega=\prod_{i} \Omega_{i}-$ Cartesian product of subsets $\Omega_{i}$

$A=\prod_{i} A_{i}--$ multiplication of the respective

$\sigma$-algebra

$P=P 1^{3}=P 1 \times P 1 \times P 1$,

$P$ - probability measure that is equal to the multiplication of the probability measures (AndersonEssen Theorem [1]).

The entropy $H_{2}\left(X_{1}, X_{3}, X_{4}, X_{5}\right)$ of the random variables $X_{1}, X_{3}, X_{4}, X_{5}$ is equal to the sum of entropies of the random vector $\bar{X}^{*}\left(X_{1}, X_{3}, X_{4}\right)$, and the random variable $X_{5}$.

$$
H_{2}\left(X_{1}, X_{3}, X_{4}, X_{5}\right)=H_{0}\left(\bar{X}^{*}\right)+H_{1}\left(X_{5}\right),
$$

$\mathrm{H}_{0}\left(\overline{\mathrm{X}}^{*}\right)$ - the entropy of the random vector $\overline{\mathrm{X}}^{*}\left(\mathrm{X}_{1}, \mathrm{X}_{3}, \mathrm{X}_{4}\right)$;

$\mathrm{H}_{1}\left(\mathrm{X}_{5}\right)$ - the differential entropy of the continuous random variable $\mathrm{X}_{5}$.

$$
\begin{aligned}
& H_{0}\left(\bar{X}^{*}\right)= \\
& =-\sum_{i j k} p\left(x_{1 i}, x_{3 j}, x_{4 k}\right) \log _{2} p\left(x_{1 i}, x_{3 j}, x_{4 k}\right)
\end{aligned}
$$

Below the results of the calculations of the above entropy indicators: $H_{0}\left(\bar{X}^{*}\right)$ - the entropy of the random vector $\bar{X}^{*}\left(X_{1}, X_{3}, X_{4}\right)$ that characterises the structural entropy of the corporate financial ratios; $H_{1}\left(X_{5}\right)$ - the entropy of the random variable $X_{5}$, which is the corporate financial attractiveness indicator (ROC). 
Table 1

\begin{tabular}{||c|c|c|c||}
\hline \hline $\begin{array}{c}\text { ENTROPY } \\
\text { (BIT) }\end{array}$ & $\boldsymbol{H}_{\mathbf{0}}$ & $\boldsymbol{H}_{\mathbf{1}}$ & $\boldsymbol{H}_{\mathbf{2}}$ \\
\hline LUKOIL & 1.21 & -0.43 & 0.78 \\
\hline MTS & 2.04 & -0.48 & 1.56 \\
\hline ROSTELECOM & 1.88 & -0.53 & 1.35 \\
\hline
\end{tabular}

\section{Standard Distribution Entropy Maximization}

There is a difference between the structural entropy indicators $H_{0}$ of the above companies. Smaller deviation of the entropy indicator means a better financial position of a selected company.

The $\mathrm{H}_{2}$ is the sum of the above entropy indicators and can be used as an integrated numerical indicator of the company`s investment attractiveness. Note that the maximum values of entropy $h(\varphi)$ of the random variable $X_{5}$ are calculated (Table 1 ).

$$
\begin{gathered}
H_{1}\left(X_{5}\right)=-\int_{\Omega_{5}} p(x) \log p(x) d x, \\
H_{1}\left(X_{5}\right) \leq h(\varphi)
\end{gathered}
$$

Three types of the maximum values of entropy of the random variable $X_{5}$, which is the corporate financial attractiveness indicator (ROC), are determined by the author in [7].

Suppose that $X_{5}$ are stock prices time series and are random processes with their own probability distribution. Then special probability distributions, approximately coinciding with the statistical ones for the first two moments, can set the boundary of the maximum entropy of $H_{1}\left(X_{5}\right)$.

In the paper, the possibility of using three maximum entropy values is examined. The maximum entropy values are calculated on the basis of three widely used probability distributions: exponential, normal and log-normal.

The theorems of differential entropy maximization for standard distributions are proved by means of the Kullback - Leibler metric. This metric is used as an informational measure of the difference between two probability distributions defined by probability densities.

In this paper, the random variable $X_{5}$ was approximated by normal and log-normal distributions. In this case, the values of the maximum differential entropy are calculated on the basis of statistical estimates for the moments of the corresponding probability distributions (Table 2).
Table 2

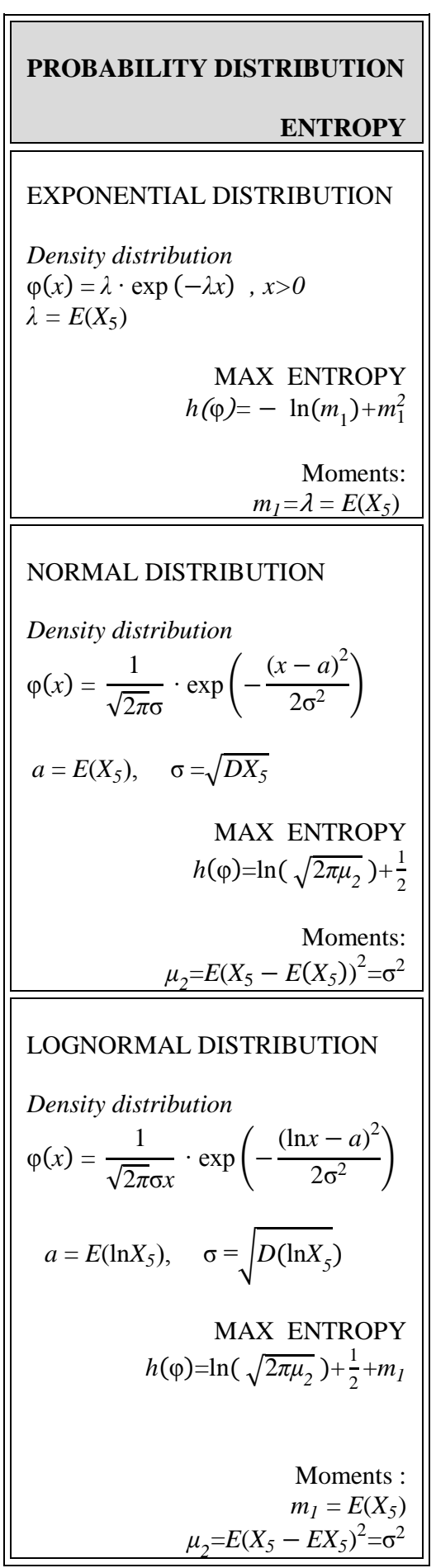

\section{Conclusion}

Thus, the entropy indicators can be applied to the integrated numerical evaluation of the company`s investment attractiveness. The corporate financial attractiveness can be evaluated on the basis of the selected significant factors, i.e. financial ratios and market ratios.

The uncertainty of the corporate financial attractiveness can be assessed using the entropy indicator of a random vector. The entropy indicators are defined 
by the use of probability measures. Discrete entropy models define the structural entropy of the corporate financial ratios. Differential entropy models are used to evaluate the corporate financial attractiveness indicator (ROC).

In accordance with the maximum entropy principle, the higher the entropy, the less "restrictions" are imposed on the system.

Transition of the system from a low-entropy state to a high-entropy state occurs when there is a gradual decrease in the influence of "restrictions" on the system. For example, when calculating differential entropy of log-normal and normal distributions, the following takes place. If the number of random events increases, a log-normal distribution with two moment constraints becomes a distribution with one "constraint", i.e. the dispersion-constraint (Table 2). In this case, the asymmetry disappears, and the distribution approximates to normal distribution. The maximum entropy formula, containing only one statistical moment (logarithmic dispersion), is used for log-normally distributed objects of a physical nature [13]. The author presents the maximum entropy values of the basic statistical distributions (Table 2), using the theorems from [7].

In a polemical aspect, it is worth noting that the maximum entropy for a log-normal distribution can be defined more accurately by the use of two statistical moments.

Thus, the obtained estimates of the maximum entropy make it possible to estimate the upper bound of the entropy of the indicator Rate of Change indicator (ROC).

As a result, in [11, 12], when assessing the risks of investment projects, the entropy indicator was used instead of the dispersion.

\section{References}

1. A.N. Kolmogorov, Selected works. Information theory and theory of algorithms (Nauka, Moscow, 1987) [in Russian]

2. Claude E. Shannon, Bell System Technical J., 27 (3), 379-423 (1948)

3. A.N. Burenin, Securities and derivatives market (S.I. Vavilov NTO, Moscow, 2015) [in Russian]

4. D. Kennedy, Stochastic Financial Models (Chapman\& Hall / CRC Finance Mathematics series, Douglas, 2010)

5. A. Gjendemsjø(ed.), Information and Signal Theory (Rice University, Houston, 2006)

6. T.V. Ryzhkova, Vestnik of MSTU “Stankin”, 2(20), 124-127 (2012) [in Russian]

7. T.V. Ryzhkova, Fundamental physical and mathematical problems and modeling of technical and technological systems, 17, 238-249 (2016) [in Russian]

8. Aksenov, P.O. Amblard, O. Michel, C. Jutten, 13th International Conference on Latent Variable
Analysis and Signal Separation (Springer, Cham, 509-518, 2017)

9. E.A. Gevorkyan, A.V. Sinchukov, O.V. Tatarnikov, Fundamental research J., 6, 121-126 (2017) [in Russian]

10. I.V. Sukhorukova, N.A. Chistiakova, Journal of Reviews on Global Economics, 7, 146-151 (2018)

11. E.V. Bozhenko, T.Y. Makhina, Journal of Economic Studies, 4(3), 30-38 (2018) [in Russian]

12. E.V. Bozhenko, Fundamental physical and mathematical problems and modeling of technical and technological systems, 17, 45-53 (2016) [in Russian]

13. B.V. Karasev, American institute of Physics, 284287 (1983)

14. A.E. Karlik, V.V. Platonov, M.V. Tihonova, E.A. Jakovleva, MIR (Modernization. Innovation. Research), 10(3), 380-394 (2019) [in Russian] 